\title{
$n \times$ OVERSAMPLING PRESERVES ANY TIGHT AFFINE FRAME FOR ODD $n$
}

\author{
CHARLES K. CHUI AND XIANLIANG SHI
}

(Communicated by J. Marshall Ash)

\begin{abstract}
If $\psi$ generates an affine frame $\psi_{j, k}(x)=2^{j / 2} \psi\left(2^{j} x-k\right), j, k \in$ $\mathbb{Z}$, of $L^{2}(\mathbb{R})$, we prove that $\left\{n^{-1 / 2} \psi_{j, k / n}\right\}$ is also an affine frame of $L^{2}(\mathbb{R})$ with the same frame bounds for any positive odd integer $n$. This establishes the result stated as the title of this paper. A counterexample of this statement for $n=2$ is also given.
\end{abstract}

\section{INTRODUCTION AND RESULTS}

Let $L^{2}=L^{2}(\mathbb{R})$ denote, as usual, the space of all complex-valued squareintegrable functions on the real line with inner product $\langle$,$\rangle and norm \|\|$. For any $f \in L^{2}$, we will use the notation

$$
f_{j, \alpha}(x)=2^{j / 2} f\left(2^{j} x-\alpha\right), \quad j \in \mathbb{Z}, \alpha \in \mathbb{R} .
$$

A function $\psi \in L^{2}$ is said to generate an affine frame

$$
\left\{\psi_{j, k}: j, k \in \mathbb{Z}\right\}
$$

of $L^{2}$, with frame bounds $A$ and $B$, where $0<A \leq B<\infty$, if it satisfies

$$
A\|f\|^{2} \leq \sum_{j, k \in \mathbb{Z}}\left|\left\langle f, \psi_{j, k}\right\rangle\right|^{2} \leq B\|f\|^{2}, \quad f \in L^{2} .
$$

The frame (1.2) of $L^{2}$ is called a tight frame, if (1.3) holds with $A=B$. The importance of a tight frame is that any $f \in L^{2}$ can be recovered from its integral wavelet transform (IWT)

$$
\left\langle f, \psi_{j, k}\right\rangle=2^{j / 2} \int_{-\infty}^{\infty} f(x) \overline{\psi\left(2^{j}\left(x-\frac{k}{2^{j}}\right)\right)} d x
$$

relative to $\psi$ at the time-scale locations $\left(2^{-j}, k / 2^{j}\right), j, k \in \mathbb{Z}$, via the formula

$$
f(x)=\frac{1}{A} \sum_{j, k \in \mathbb{Z}}\left\langle f, \psi_{j, k}\right\rangle \psi_{j, k}(x),
$$

Received by the editors March 11, 1992 and, in revised form, September 22, 1992.

1991 Mathematics Subject Classification. Primary 41A17, 41A58, 42C99.

Key words and phrases. Frames, frame bounds, wavelets, oversampling, recovery from wavelet transforms.

This research was supported by NSF Grant DMS-89-01345 and ARO Contract DAAL 03-90-G0091 . 
where $A=B$. It should be noted that a frame, such as $(1.2)-(1.3)$, tight or not, may be redundant in the sense that (1.2) does not have to be $l^{2}$-linearly independent. However, any Riesz (or unconditional) basis is also a frame.

In the above discussion, we only consider, without loss of generality, the sampling period $b=1$ and scaling parameter $a=2$ as in (1.2). Details and generality are discussed in the wavelet literature $[1,2,3,5,6]$, and a general study of frames can be found in the monograph [7] as well as the fundamental paper [4] of Duffin and Schaeffer, where the notion of frames was first introduced.

The objective of this paper is to establish the following.

Theorem 1. Let $\psi \in L^{2}$ generate a frame $\left\{\psi_{j, k}\right\}$ of $L^{2}$ with frame bounds $A$ and $B$ as given by (1.3). Then for any positive odd integer $n$, the family

$$
\left\{n^{-1 / 2} \psi_{j, k / n}: j, k \in \mathbb{Z}\right\}
$$

remains a frame of $L^{2}$ with the same bounds: that is,

$$
n A\|f\|^{2} \leq \sum_{j, k \in \mathbb{Z}}\left|\left\langle f, \psi_{j, k / n}\right\rangle\right|^{2} \leq n B\|f\|^{2}, \quad f \in L^{2} .
$$

In particular, if $\left\{\psi_{j, k}\right\}$ is a tight frame (with $A=B$ ) and $n>0$ is odd, then the family in (1.6) satisfies

$$
\sum_{j, k \in \mathbb{Z}}\left|\left\langle f, \psi_{j, k / n}\right\rangle\right|^{2}=n A\|f\|^{2}, \quad f \in L^{2} .
$$

On the other hand, (1.8) does not necessarily hold for even $n>0$.

\section{Preliminary Results}

A sequence of three lemmas will be needed for the proof of Theorem 1. Let $n$ be any positive odd integer and set

$$
\lambda_{1}(p)=2 p-\frac{n}{2}(1+\operatorname{sgn}(2 p-n)) .
$$

Then $\lambda_{1}$ is a permutation of the set $\{0, \ldots, n-1\}$. This permutation gives rise to a rearrangement operator $\tau$ defined on $\mathbb{R}^{n}$ by

$$
\tau(\mathbf{a}):=\left(a_{\lambda_{1}(0)}, \ldots, a_{\lambda_{1}(n-1)}\right), \quad \mathbf{a}=\left(a_{0}, \ldots, a_{n-1}\right) \in \mathbb{R}^{n},
$$

where $n>0$ is odd. As usual, we set

$$
\tau^{0}=I \quad \text { and } \quad \tau^{j}=\tau\left(\tau^{j-1}\right), \quad j=1,2, \ldots,
$$

with $I$ denoting the identity operator. Hence, for each $j=0,1, \ldots$, we may write

$$
\tau^{j}(\mathbf{a})=\left(a_{\lambda_{j}(0)}, \ldots, a_{\lambda_{j}(n-1)}\right), \quad \mathbf{a} \in \mathbb{R}^{n},
$$

where $\lambda_{j}$ is a permutation of $\{0, \ldots, n-1\}$ induced by $\lambda_{1}$. We have the following result. 
Lemma 1. Let $n$ be any positive odd integer and $\lambda_{j}$ be defined by (2.1)-(2.4). Then for any $j \in \mathbb{Z}_{+}$,

$$
\lambda_{j}(p) \equiv 2^{j} p \quad(\bmod n), \quad p=0, \ldots, n-1 .
$$

Proof. We will establish (2.5) by induction on $j$. Since $\lambda_{0}$ is the identity, (2.5) certainly holds for $j=0$. For $j \geq 1$, we first consider $0 \leq p \leq(n-1) / 2$. In this case, it follows from $(2.1)$ that $\lambda_{j+1}(p)=\lambda_{j}(2 p)$. Hence, by the induction hypothesis, we have

$$
\lambda_{j+1}(p)=\lambda_{j}(2 p) \equiv 2^{j}(2 p)(\bmod n)=2^{j+1} p(\bmod n)
$$

Similarly, for $(n-1) / 2<p \leq n-1$, it follows from $(2.1)$ and the induction hypothesis that

$$
\lambda_{j+1}(p)=\lambda_{j}(2 p-n) \equiv 2^{j}(2 p-n)(\bmod n) \equiv 2^{j+1} p(\bmod n) .
$$

Since $\lambda_{1}$ is a one-one map of $\{0, \ldots, n-1\}$ onto itself, the rearrangement operator $\tau$ as defined by (2.2) has an inverse $\tau^{-1}$. Hence, the definition of $\tau^{j}$ in (2.3) can be extended to all $j \in \mathbb{Z}$. Set

$$
\mathbf{a}_{0}:=(0,1, \ldots, n-1)
$$

and define $\left\{\varepsilon_{j, p}\right\}, p=0, \ldots, n-1$, and $j \in \mathbb{Z}$, by

$$
\tau^{j}\left(\mathbf{a}_{0}\right):=\left(\varepsilon_{j, 0}, \ldots, \varepsilon_{j, n-1}\right), \quad j \in \mathbb{Z} .
$$

We have the following

Lemma 2. Let $j_{0} \in \mathbb{Z}$. Then

$$
\varepsilon_{j, p} \equiv 2^{j-j_{0}} \varepsilon_{j_{0}, p} \quad(\bmod n),
$$

for all $j>j_{0}$ and $p=0, \ldots, n-1$.

Proof. We first establish the relation:

$$
\varepsilon_{j+1, p} \equiv 2 \varepsilon_{j, p} \quad(\bmod n), \quad j \leq-1, p=0, \ldots, n-1,
$$

by induction. For $j=-1$, observe that for even $p$,

$$
2 \varepsilon_{-1, p}=2 \varepsilon_{0, p / 2} \equiv p \quad(\bmod n),
$$

and that for odd $p$,

$$
2 \varepsilon_{-1, p}=2 \varepsilon_{0,[p / 2]+(n+1) / 2}=2[p / 2]+n+1 \equiv p \quad(\bmod n) .
$$

Hence, (2.8) holds for $j=-1$. For $j<-1$, we have, for even $p$,

$$
2 \varepsilon_{j, p}=2 \varepsilon_{j+1, p / 2} \equiv \varepsilon_{j+2, p / 2} \quad(\bmod n)
$$

by applying the induction hypothesis. Since $\varepsilon_{j+2, p / 2}=\varepsilon_{j+1, p}$, it follows that

$$
2 \varepsilon_{j, p} \equiv \varepsilon_{j+1, p} \quad(\bmod n),
$$

for even $p$. For odd $p$, we also have

$$
\begin{aligned}
2 \varepsilon_{j, p} & =2 \varepsilon_{j+1,[p / 2]+(n+1) / 2} \equiv \varepsilon_{j+2,[p / 2]+(n+1) / 2}(\bmod n) \\
& \equiv \varepsilon_{j+1, p}(\bmod n)
\end{aligned}
$$

again by applying the induction hypothesis. This establishes (2.8). 
Of course, (2.7) is an immediate consequence of (2.8) for $0 \geq j>j_{0}$. On the other hand, if $j>0>j_{0}$, then we may obtain (2.7) by applying (2.5) in Lemma 1 as well. Finally, for $j>j_{0} \geq 0$, then

$$
\varepsilon_{j, p} \equiv 2^{j} \varepsilon_{0, p}(\bmod n) \equiv 2^{j-j_{0}} 2^{j_{0}} \varepsilon_{0, p}(\bmod n) \equiv 2^{j-j_{0}} \varepsilon_{j_{0}, p}(\bmod n) .
$$

Lemma 3. Let $j, j_{0} \in \mathbb{Z}$ with $j \geq j_{0}$ and $p=0, \ldots, n-1$. Then the two collections of functions

$$
\left\{2^{j} x-\varepsilon_{j, p} / n-k: k \in \mathbb{Z}\right\}
$$

and

$$
\left\{2^{j}\left(x-2^{-j_{0}} \varepsilon_{j_{0}, p} / n\right)-k^{\prime}: k^{\prime} \in \mathbb{Z}\right\}
$$

are identical.

Proof. Let $k \in \mathbb{Z}$. Since $\varepsilon_{j, k} \equiv 2^{j-j_{0}} \varepsilon_{j_{0}, p}(\bmod n)$, we have

$$
2^{j} x-\frac{\varepsilon_{j, p}}{n}-k=2^{j} x-\frac{2^{j-j_{0}} \varepsilon_{j_{0}, p}}{n}-k^{\prime}=2^{j}\left(x-\frac{2^{-j_{0}} \varepsilon_{j_{0}, p}}{n}\right)-k^{\prime}
$$

for some $k^{\prime} \in \mathbb{Z}$. In addition, it is quite easy to see that the mapping $k \rightarrow k^{\prime}$ is one-to-one. Hence, the two collections (2.9) and (2.0) are identical.

\section{Proof of Theorem 1}

Let $n$ be a positive odd integer. We first decompose the collection of functions $\psi_{j, k / n}, j, k \in \mathbb{Z}$, into $n$ disjoint subcollections $S_{0}, \ldots, S_{n-1}$, where

$$
S_{p}:=\left\{2^{j / 2} \psi\left(2^{j} x-\varepsilon_{j, p} / n-k\right): j, k \in \mathbb{Z}\right\} .
$$

Since $S_{0}$ is the set $\left\{\psi_{j, k}: j, k \in \mathbb{Z}\right\}$, the assumption (1.3) can be expressed as

$$
A\|f\|^{2} \leq \sum_{g \in S_{0}}|\langle f, g\rangle|^{2} \leq B\|f\|^{2}, \quad f \in L^{2} .
$$

Let $j_{0} \in \mathbb{Z}$ and consider

$$
\sigma_{j_{0}, p}(f):=\sum_{j \geq j_{0}} \sum_{k \in \mathbb{Z}}\left|\left\langle 2^{j / 2} \psi\left(2^{j} \cdot \frac{\varepsilon_{j, p}}{n}-k\right), f\right\rangle\right|^{2},
$$

where $p=0, \ldots, n-1$, and $f \in L^{2}$. By Lemma 3, we see that

$$
\begin{aligned}
\sigma_{j_{0}, p}(f) & =\sum_{j \geq j_{0}} \sum_{k \in \mathbb{Z}}\left|\left\langle 2^{j / 2} \psi\left(2^{j}\left(\cdot-\frac{2^{-j_{0}} \varepsilon_{j_{0}, p}}{n}\right)-k\right), f\right\rangle\right|^{2}, \\
& =\sum_{j \geq j_{0}} \sum_{k \in \mathbb{Z}}\left|\left\langle 2^{j / 2} \psi\left(2^{j} \cdot-k\right), f\left(\cdot+\frac{2^{-j_{0}} \varepsilon_{j_{0}, p}}{n}\right)\right\rangle\right|^{2} \\
& \leq B\left\|f\left(\cdot+\frac{2^{-j_{0}} \varepsilon_{j_{0}, p}}{n}\right)\right\|^{2}=B\|f\|^{2}
\end{aligned}
$$

Hence, for each $p=1, \ldots, n-1$, we have

$$
\sum_{g \in S_{p}}|\langle f, g\rangle|^{2}=\lim _{j_{0} \rightarrow-\infty} \sigma_{j_{0}, p}(f) \leq B\|f\|^{2} .
$$


Combining this with (3.3) yields

$$
\sum_{j, k \in \mathbf{Z}}\left|\left\langle f, \psi_{j, k / n}\right\rangle\right|^{2}=\sum_{p=0}^{n-1} \sum_{g \in S_{p}}|\langle f, g\rangle|^{2} \leq n B\|f\|^{2} .
$$

To establish the lower bound in (1.7) we consider the class $L_{c}^{\infty}$ of all a.e. bounded functions with compact support in $\mathbb{R}$. Since $L_{c}^{\infty}$ is dense in $L^{2}$, it is sufficient to prove that the lower bound in (3.3) holds for all $f \in L_{c}^{\infty}$. Let $f \in L_{c}^{\infty}$ and suppose that

$$
\operatorname{supp} f \subset[-L, L], \quad L>0 .
$$

Set

$$
\boldsymbol{\Theta}_{j_{0}, p}(f):=\sum_{j \geq j_{0}} \sum_{k \in \mathbf{Z}}\left|\left\langle 2^{j / 2} \psi\left(2^{j} \cdot-k\right), f_{j_{0}}^{p}\right\rangle\right|^{2}
$$

and

$$
\Lambda_{j_{0}, p}(f):=\sum_{j<j_{0}} \sum_{k \in \mathbf{Z}}\left|\left\langle 2^{j / 2} \psi\left(2^{j} \cdot-\frac{\varepsilon_{j, p}}{n}-k\right), f\right\rangle\right|^{2}
$$

where

$$
f_{j_{0}}^{p}:=f\left(x+2^{-j_{0}} \varepsilon_{j_{0}, p} / n\right) .
$$

By (3.2), we have, for each $p=1, \ldots, n-1$,

$$
\sigma_{j_{0}, p}(f)+\boldsymbol{\Theta}_{j_{0}, p}(f)=\sum_{j, k \in \mathbf{Z}}\left|\left\langle\psi_{j, k}, f_{j_{0}}^{p}\right\rangle\right|^{2} \geq A\left\|f_{j_{0}}^{p}\right\|^{2}=A\|f\|^{2} .
$$

This yields

$$
\begin{aligned}
\sum_{\boldsymbol{g} \in S_{p}}|\langle f, g\rangle|^{2} & =\sum_{j, k \in \mathbf{Z}}\left|\left\langle 2^{j / 2} \psi\left(2^{j} \cdot-\frac{\varepsilon_{j, p}}{n}-k\right), f\right\rangle\right|^{2} \\
& =\sigma_{j_{0}, p}(f)+\boldsymbol{\Theta}_{j_{0}, p}(f)-\boldsymbol{\Theta}_{j_{0}, p}(f)+\Lambda_{j_{0}, p}(f) \\
& \geq A\|f\|^{2}-\boldsymbol{\Theta}_{j_{0}, p}(f) .
\end{aligned}
$$

By introducing the notation

$$
I_{j_{0}, p}:=\left[-L-2^{-j_{0}} \varepsilon_{j_{0}, p} / n, L-2^{-j_{0}} \varepsilon_{j_{0}, p} / n\right],
$$

it follows from (3.5) and (3.8) that

$$
\operatorname{supp} f_{j_{0}}^{p} \subset I_{j_{0}, p}, \quad p=1, \ldots, n-1 .
$$

Hence, by the Cauchy inequality, we have

$$
\begin{aligned}
\left|\left\langle 2^{j / 2} \psi\left(2^{j} \cdot-k\right), f_{j_{0}}^{p}\right\rangle\right|^{2} & \leq 2^{j+1} L\|f\|_{\infty} \int_{I_{j_{0}, p}}\left|\psi\left(2^{j} x-k\right)\right|^{2} d x \\
& =2 L\|f\|_{\infty} \int_{2^{j} I_{j_{0}, p}}|\psi(x-k)|^{2} d x .
\end{aligned}
$$


Let $j_{0}, J \in \mathbb{Z}$ be so chosen that $j_{0}<-\log _{2} n L$ and $J>\log _{2}(L+1)$. Then $I_{j_{0}, p} \subset(-\infty, 0)$, and hence

$$
\begin{aligned}
\Theta_{j_{0}, p}(f) \leq & 2 L\|f\|_{\infty} \sum_{j_{0}-J \leq j<j_{0}} \sum_{k \in \mathbb{Z}} \int_{k-2^{j} L-2^{-j_{0}+j} \varepsilon_{j_{0}, p} n^{-1}}^{k+2^{j} L-2^{-j_{0}+j} \varepsilon_{j_{0}, p} n^{-1}}|\psi(x)|^{2} d x \\
& +2 L\|f\|_{\infty} \sum_{k \in \mathbb{Z}} \int_{k-2^{-J} L-2^{-J}(n-1) n^{-1}}^{k}|\psi(x)|^{2} d x .
\end{aligned}
$$

Let $\eta>0$ be arbitrarily given. Since $\psi \in L^{2}$, there is some $\beta>0$ such that $\int_{|x| \geq \beta}|\psi(x)|^{2} d x<\eta$. So, by setting $\psi_{\beta}:=\psi \chi_{[-\beta, \beta]}, \beta>0$, we have

$$
\begin{array}{r}
\boldsymbol{\Theta}_{j_{0}, p} \leq 2 L\|f\|_{\infty}\left(\eta+\sum_{j_{0}-J \leq j<j_{0}} \sum_{k \in \mathbb{Z}} \int_{k-2^{j} L-2^{-j_{0}{ }^{+j} \varepsilon_{j_{0}, p} n^{-1}}}^{k+2^{j} L-2^{-j^{+j}} \varepsilon_{j_{0}, p} n^{-1}}|\psi(x)|^{2} d x\right. \\
\left.+\sum_{k \in \mathbb{Z}} \int_{k-2^{-J} L-2^{-J}(n-1) n-1}^{k}\left|\psi_{\beta}(x)\right|^{2} d x\right)
\end{array}
$$

where the last term on the right-hand side is smaller than $\eta$ for any sufficiently large $J$. For such a fixed $J$, a $\gamma>0$ can be chosen to yield

$$
\int_{|x| \geq \gamma}|\psi(x)|^{2} d x \leq \eta J^{-1}
$$

Hence, it follows from (3.10) that

$$
\boldsymbol{\Theta}_{j_{0}, p}(f) \leq 2 L\|f\|_{\infty}\left(3 \eta+\sum_{j_{0}-J \leq j \leq j_{0}} \sum_{k \in \mathbb{Z}} \int_{k-2^{j} L-2^{-j_{0} j_{\varepsilon_{j}, p} n^{-1}}}^{k+2^{j} L-2^{-j_{0}+j_{j}} \varepsilon_{j_{0}, p} n^{-1}}\left|\psi_{\gamma}(x)\right|^{2} d x\right),
$$

where (3.11) has been used to take care of $\psi-\psi_{\gamma}$. Since $\psi_{\gamma}$ has compact support, the last term on the right-hand side of (3.12) tends to zero as $j_{0} \rightarrow-\infty$, so that

$$
\limsup _{j_{0} \rightarrow-\infty} \Theta_{j_{0}, p}(f) \leq 6 L\|f\|_{\infty} \eta, \quad p=1, \ldots, n-1 .
$$

In view of (3.9) and (3.2), we have established the lower bound in (1.7).

Finally, to show that (1.8) does not necessarily hold for even $n>0$, we consider two functions

$$
f_{1}(x)=\psi_{H}\left(x+\frac{1}{2}\right) \quad \text { and } \quad f_{2}(x)=\chi_{[-1 / 2,1 / 2)}(x),
$$

where $\psi_{H}(x)=\chi_{[0,1)}(x) \operatorname{sgn}\left(\frac{1}{2}-x\right)$ is the Haar function. Then for $\psi=\psi_{H}$ in (1.8), we have

$$
\sum_{j, k \in \mathbb{Z}}\left|\left\langle f_{1}, \psi_{H ; j, k / 2}\right\rangle\right|^{2}=3 \sum_{j, k \in \mathbb{Z}}\left|\left\langle f_{2}, \psi_{H ; j, k / 2}\right\rangle\right|^{2}=\frac{9}{2},
$$

while $\left\|f_{1}\right\|=\left\|f_{2}\right\|=1$. Hence, $\left\{\psi_{H ; j, k / 2}\right\}$ cannot be a tight frame. 


\section{REFERENCES}

1. C. K. Chui, An introduction to wavelets, Academic Press, Boston, 1992.

2. I. Daubechies, The wavelet transform, time-frequency localization and signal analysis, IEEE Trans. Inform. Theory 36 (1990), 961-1005.

3. I. Daubechies, Ten lectures on wavelets, CBMS-NSF Regional Conf. Ser. in Appl. Math., vol. 61, SIAM, Philadelphia, PA, 1992.

4. R. J. Duffin and A. C. Schaefer, A class of nonharmonic Fourier series, Trans. Amer. Math. Soc. 72 (1952), 341-366.

5. C. Heil and D. Walnut, Continuous and discrete wavelet transforms, SIAM Rev. 31 (1989), 628-666.

6. Y. Meyer, Ondelettes et opérateurs, Vol. 1, Hermann, Paris, 1990.

7. R. M. Young, An introduction to nonharmonic Fourier series, Academic Press, New York, 1980.

Department of Mathematics, Texas A\&M University, College Station, Texas 778433368

Permanent address, Xianliang Shi: Department of Mathematics, Hangzhou University, Hangzhou, People's Republic of China

E-mail address, Charles K. Chui: cat@math.tamu.edu

E-mail address, Xianliang Shi: shi@wavelet1.math.tamu.edu 